\title{
A micro-peptide encoded by HOXB-AS3 promotes the proliferation and viability of oral squamous cell carcinoma cell lines by directly binding with IGF2BP2 to stabilize c-Myc
}

\author{
FEI LENG, YAN-YU MIU, YI ZHANG, HAO LUO, XIAO-LI LU, \\ HUI CHENG and ZHI-GUO ZHENG \\ The Affiliated Stomatological Hospital of Nanchang University, The Key Laboratory \\ of Oral Biomedicine, Nanchang, Jiangxi 330006, P.R. China \\ Received April 17, 2021; Accepted June 18, 2021
}

DOI: $10.3892 / \mathrm{ol} .2021 .12958$

\begin{abstract}
HOXB-AS3 is a long non-coding RNA and recent studies have shown that the HOXB-AS3-encoded micro-peptide was associated with the progression of colon cancer tumorigenesis; however, the biofunction of HOXB-AS3 varies in different types of cancer and the potential function in oral squamous cell carcinoma (OSCC) is still unknown. The Cancer Genome Atlas (TCGA) database was searched and the expression patterns of HOXB-AS3 in head and neck carcinoma were analyzed. Reverse transcription-quantitative PCR and western blot analysis was used to measure the mRNA and protein expression level of HOXB-AS3 in patients with OSCC, respectively. Next, HOXB-AS3 was knocked down in 2 OSCC cell lines to investigate the biological function of the HOXB-AS3-encoded protein using a Cell Counting Kit-8 and colony formation assays. To further identify the potential mechanism of the HOXB-AS3-encoded protein, co-immunoprecipitation was also used to detect the interaction between HOXB-AS3 and IGF2BP2, while HOXB-AS3 was re-expressed to determine whether the HOXB-AS3-encoded protein and not HOXB-AS3 exerted its function in OSCC. HOXB-AS3 was upregulated in OSCC tissues, in both TCGA database and in patients with OSCC recruited into the present study. HOXB-AS3 was associated with poor prognosis in OSCC. The proliferation and viability decreased in the 2 OSCC cell lines following knock down of HOXB-AS3. HOXB-AS3 was also found to encode a protein that directly interacted with IGF2BP2 and thereby promoted the stability of c-myc. Taken together, the results from the present study indicated
\end{abstract}

Correspondence to: Professor Zhi-Guo Zheng, The Affiliated Stomatological Hospital of Nanchang University, The Key Laboratory of Oral Biomedicine, 49 Fuzhou Road, Nanchang, Jiangxi 330006, P.R. China

E-mail: zhengzhiguo666@126.com

Key words: IncRNA, HOXB-AS3, OSCC, proliferation, c-Myc, IGF2BP2 that increased HOXB-AS3 expression was associated with poor prognosis in OSCC. This indicated that HOXB-AS3 and its encoded protein promoted OSCC cell proliferation and viability by maintaining c-Myc mRNA stability by directly binding to IGF2BP2.

\section{Introduction}

Oral cancer is the 6th most common malignancy worldwide and oral squamous cell carcinoma (OSCC) represents $90 \%$ of all cancer cases (1). The current therapy is only effective for early stage OSCC, and the 5-year overall survival rate was $<50 \%$ in 2020 (2). Studies have indicated that tobacco addiction, excessive alcohol consumption and human papillomavirus (HPV) infection are the main risk factors for tumorigenesis and the development of OSCC (3). Current targeted therapies, such as targeting PD1/PDL1, are far from satisfactory (4); therefore; new predictive biomarkers and therapeutic targets are urgently required.

Long non-coding (lnc)RNAs are 200 nucleotides in length (5). With the application of high-throughput sequencing, lncRNAs have been reported to be associated with the progression of numerous types of cancer. Rs6695584 single nucleotide polymorphism-induced lncRNA-SLCC1 drove colorectal cancer by activating glycolysis signaling (6). lncRNA lnc030 maintained breast cancer stemness by stabilizing SQLE mRNA and therefore promoted cholesterol synthesis (7). IncRNA CRNDE attenuated chemoresistance in gastric cancer by SRSF6-regulated alternative splicing of PICALM (8). HOXB-AS3 was previously reported to encode a micro-peptide and to suppress tumor progression in colon cancer (9). However, biofunction varies in different types of cancer $(10,11)$. Its potential function and underlying mechanism in OSCC are still unknown.

In the present study, the expression level of HOXB-AS3 in patients with OSCC was analyzed and its biological functions and underlying mechanism in OSCC cell lines was also investigated. The results demonstrated, to the best of knowledge, for the first time that HOXB-AS3 and its encoded protein were upregulated in OSCC tumors. Knocking down HOXB-AS3 repressed OSCC cell proliferation and viability, 
and a Co-immunoprecipitation (IP) assay indicated that the HOXB-AS3-encoded protein interacted with IGF2BP2; therefore, stabilized c-Myc.

\section{Materials and methods}

Patient information and clinical sample collection. Paired OSCC samples and adjacent normal tissues were randomly collected from patients who underwent resection surgery at the Affiliated Stomatological Hospital of Nanchang University (Jiangxi, China) between January 2012 and December 2013. The following inclusion criteria was used: Diagnosed with OSCC by pathologists between January 2012 and December 2013 at the Affiliated Stomatological Hospital of Nanchang University. Patients with metastasis were excluded. A total of 20 cases (age range, 42-68 years; 15 male and 5 female) were divided into 4 groups, according to their clinical features and the TNM stage system (12). There were 5 patients with stage I, 3 patients with stage II, 8 patients with stage III and 4 cases with stage 4 . Adjacent normal tissues were collected $>5 \mathrm{~cm}$ from the tumor site and confirmed by a pathologist as normal tissue. All the OSCC tissue samples were confirmed by pathology. The present study was approved by the Ethics Committee of The Affiliated Stomatological Hospital of Nanchang University (Jiangxi, China). The clinical samples were collected from patients following written informed consent.

Survival analysis. Using data from The Cancer Genome Atlas (TCGA) database, the time of the end point of each patient was collected, this was defined as the survival end point of each patient, all the survival end points in of the whole cohort was defined as overall survival. The overall survival analysis was thus automatically determined using the Gene Expression Profiling Interactive Analysis online tool by analyzing these data (gepia.cancer-pku.cn) by inputing the gene name (HOXB-AS3) and cancer type (OSCC), 519 patients were enrolled and 44 normal tissues were collected. For the patients recruited into the present study, the survival time was collected between 2012 and 2018. From the patients recruited into the present study, they were divided into 2 groups according to the mean mRNA expression (3.56) and Kaplan-Meier survival analysis was performed and the GraphPad software v.7.0 (GraphPad Software, Inc.).

Reverse transcription-quantitative analysis ( $R T-q P C R)$ analysis. Tissues and cells were harvested and lysed using TRIzol $^{\circledR}$ (cat. no. 93289; Sigma-Aldrich; Merck KGaA) following the manufacturer's protocol. After purification and reverse transcription $\left(60^{\circ} \mathrm{C}\right.$ for $5 \mathrm{~min}, 37^{\circ} \mathrm{C}$ for $15 \mathrm{~min}$ and $85^{\circ} \mathrm{C}$ for $5 \mathrm{sec}$ ) cDNA was collected. Specific primers were used for qPCR using the SYBR-Green RT-PCR system with RT mix (cat. no. RR036B; Takara Bio, Inc.) and SYBRGreen (cat. no. K0243; Themo Fisher Scientific Inc.) with 2 step process. The following thermocycling conditions were used: Initial denaturation at $95^{\circ} \mathrm{C}$ for $5 \mathrm{sec}$, then 40 cycles at $95^{\circ} \mathrm{C}$ for $5 \mathrm{sec}, 95^{\circ} \mathrm{C}$ for $35 \mathrm{sec}$ and $60^{\circ} \mathrm{C}$ for $30 \mathrm{sec}$, then $60^{\circ} \mathrm{C}$ for $30 \mathrm{sec}$. GAPDH was used for normalization. The following primers were used: HOXB-AS3 forward, 5'-CCCTCCAAG TCCAGTAAGAAGT-3' and reverse 5'-AGATCCTAAGAG GTGCGAGTTTA-3'; and GAPDH forward, 5'-GGAGCGAGA
TCCCTCCAAAAT-3' and reverse 5'-GGCTGTTGTCATACT TCTCATGG-3' and the expression level was normalized to the control (13).

Cell culture and transfection. The Cal-27 and UM2 cell lines were gifts from Professor Wang from the Department of Oral and Maxillofacial Surgery, Affiliated Hospital of Jiangxi University of Traditional Chinese Medicine (Jiangxi, China). The cells were cultured with complete DMEM (cat. no. D0697; Sigma-Aldrich; Merck KGaA) containing 10\% fetal bovine serum (cat. no. F8687; Invitrogen; Thermo Fisher Scientific, Inc.) and $1 \%$ penicillin/streptomycin (cat. no. V900929; Sigma-Aldrich; Merck KGaA).

To establish a stable cell line, lentivirus was obtained from GenScript, and the following short hairpin (sh)RNAs were used in pCDH-CMV-MCS-EF1 + Puro (SBI pCD513B-1): Scrambled negative control (NC) 5'-GTACCTACGTA-3', shRNA1, 5'-CCATCCAAGTCTA-3' and shRNA2, 5'-TAA TGCAGCGTAAG-3'. ORF, the open read frame of HOXB-AS3 encoding the protein but not the whole lncRNA. Plasmids $(4 \mu \mathrm{g})$ were co-transfected with packaging vectors psPAX2 (Addgene, Inc.) $(1 \mu \mathrm{g})$ and pMD2G (Addgene, Inc.) (3 $\mu \mathrm{g})$ into 293T (cat. no. CRL-11268; ATCC) cells for lentivirus production using Lipofectamine $3000^{\circledR}$ (Thermo Fisher Scientific Inc.) in accordance to the manufacturer's instructions. After $72 \mathrm{~h}$, the suspension was collected. The lentivirus was stored at $1 \times 10^{8} / \mathrm{ml}$. The cells were seeded into the 6 -well plate with $40 \%$ density and incubated overnight at $37^{\circ} \mathrm{C}$, following which $10 \mu \mathrm{l}$ (12 MOI) each lentivirus was added into the corresponding well and incubated at $37^{\circ} \mathrm{C}$ for 3 days to successfully establish the stable cell line. No selection method was used due to the high transfection rate.

Western blot analysis. The cells were harvested and washed 3 times with ice cold PBS and lysed with RIPA buffer (cat. no. 20-188; Sigma-Aldrich; Merck KGaA). The protein was quantified using the BCA kit (Beyotime Institute of Biotechnology), according to the manufacturer's protocol. Equal amounts of protein ( $40 \mu \mathrm{g} / \mathrm{lane})$ were separated using $12 \%$ SDS-PAGE, then transferred onto a PVF membrane and blocked with 5\% skimmed milk for $1 \mathrm{~h}$ at room temperature. The membranes were subsequently incubated with the primary antibodies overnight at $4^{\circ} \mathrm{C}$, washed with $0.1 \%$ TBS-Tween-20, then incubated with the donkey anti mouse/rabbit secondary antibody (1:10,000; cat. no. 5724; KPL, Inc.) for $1 \mathrm{~h}$ at room temperature. The target proteins were detected using an ECL (EMD Millipore) kit. The following primary antibodies were used: HOXB-AS3 (1:1,000; cat. no. SBSN250; Genscript), $\beta$-actin (cat. no. 3700; 1:1,000; Cell Signaling Technology, Inc.), IGF2BP2 (cat. no. ab109284; 1:1,000; Abcam), and c-Myc (cat. no. ab32072; 1:1,000; Abcam).

Co-IP assay. The Cal-27 cell line was transfected with $2 \mu \mathrm{g}$ Flag tagged HOXB-AS3 (pCDH-CMV-MCS-EF1 + Puro (SBI pCD513B-1) (cat. no. SBSN251; Genscript) using Lipofectamine $3000^{\circledR}$ (Thermo Fisher Scientific Inc.) in accordance to the manufacturer's instructions at $37^{\circ} \mathrm{C}$ for 1 day. Following incubation at $37^{\circ} \mathrm{C}$ for 3 days cells were harvested. After harvesting, cells were washed with Phosphate-Buffered Saline (cat. no. C0221A; Beyotime Institute of Biotechnology) 
the cells were lysed with $600 \mu 1$ weak RIPA (cat. no. P0013D; Beyotime Institute of Biotechnology). After incubation at $4{ }^{\circ} \mathrm{C}$ for $30 \mathrm{mins}$ the suspension was collected. After centrifuging at $6 \times 10^{3} \mathrm{~g}$ for $10 \mathrm{mins}$ at $4^{\circ} \mathrm{C}$, the sediment was removed. The $2 \mu \mathrm{g}$ flag antibody (cat. no. 14793; Cell Signaling Technology, Inc.) was added, and after incubation overnight at $4^{\circ} \mathrm{C}$, the suspension was collected, a $100 \mu \mathrm{l}$ protein $\mathrm{A}+\mathrm{G}$ beads (cat. no. P2108; Beyotime Institute of Biotechnology) were added and after rolling at room temperature for 30 mins, after centrifuging at $6 \times 10^{3} \mathrm{~g}$ for $10 \mathrm{mins}$ at $4^{\circ} \mathrm{C}$, the suspension was collected and boiled at $100^{\circ} \mathrm{C}$ for $10 \mathrm{~min}$ and subjected to western blot analysis as aforementioned. IGF2BP2 was detected.

Cell proliferation assay. A total of 500 cells per well were seeded into 96 -well plates. After incubation at $37^{\circ} \mathrm{C}$ for 3 days, CCK-8 (cat. no. ab228554; Abcam,) solution was added, after incubation at $37^{\circ} \mathrm{C}$ for $4 \mathrm{~h}$, the absorption at $450 \mathrm{~nm}$ was measured. The relative absorption of sh-1,sh-2 and ORF group (experimental group) was normalized to the control group(NC). The experiments were repeated 3 times and the data are presented as the mean \pm SD.

Colony formation assay. To measure the long-term proliferation effects, 500 cells were seeded in a 6-well plate. After incubating for 14 days, the colonies were fixed for $20 \mathrm{~min}$ with $4 \%$ paraformaldehyde and stained with crystal violet (cat. no. C0775; Sigma-Aldrich; Merck KGaA) at room temperature for $10 \mathrm{~min}$, and the number of colonies was counted under a light microscope (FV100; Olympus Corporation) manually. The assay was performed 3 times and the data are presented as the mean $\pm \mathrm{SD}$.

Statistical analysis. Data was shown in mean \pm SD, all the experiments were repeated at least 3 times. All data analysis was performed with SPSS v20.0 (IBM, Corp.). The differences between 2 groups were compared using a paired Student's t-test. For $>2$ groups, one-way ANOVA was used, followed by Tukey's post hoc test. Kaplan-Meier method were used to analyze overall survival (OS) time and the curves were compared using the log-rank test. $\mathrm{P}<0.05$ was considered to indicate a statistically significant difference.

\section{Results}

HOXB-AS3 is upregulated in human OSCC tissues and is associated with poor prognosis. RNA sequencing datasets from TCGA were analyzed and HOXB-AS3 was identified as one of the dysregulated genes in head and neck cancer, which to the best of our knowledge has not been investigated previously in head and neck cancer $(\mathrm{P}<0.05$; Fig. 1A). Next, the mRNA expression level of HOXB-AS3 was analyzed in 20 randomly selected paired adjacent normal and cancer tissues from patients recruited into the present study. The results showed that HOXB-AS3 was upregulated in cancer tissue compared with that in adjacent normal tissue $(\mathrm{P}<0.001$; Fig. 1B). The patients were subsequently divided into 4 subtypes according to TNM stage. The results indicated that patients with developed oral cancer had higher mRNA expression levels of HOXB-AS3 (P<0.001; Fig. 1B).
The Kaplan-Meier survival analysis from TCGA database is shown in Fig. S1, the overall survival showed no significance. From the patients recruited into the present study, they were divided into 2 groups (high/low expression) according to the mean mRNA expression level and the OS time was analyzed. The results showed that HOXB-AS3 was associated with poor prognosis, with patients with low expression levels having poor survival time $(\mathrm{P}<0.001$; Fig. $1 \mathrm{C})$. HOXB-AS3 was also reported to encode a protein named HOXB-AS3 (8); therefore, the protein expression level of HOXB-AS3 was analyzed in 4 randomly selected tumor and paired normal tissues. The results showed that HOXB-AS3 protein expression level was increased in the cancer tissue (Fig. 1D).

$H O X B-A S 3$ promotes the proliferation of OSCC. As aforementioned, HOXB-AS3 was found to be upregulated in OSCC cancer tissue. To identify the biofunction of HOXB-AS3 in the progression of the OSCC cell lines, HOXB-AS3 stable knockdown cell lines were established using the Cal-27 and UM2 cell lines. The relative mRNA ( $\mathrm{P}<0.001$; Fig. $2 \mathrm{~A})$ and protein (Fig. 2B) expression level of HOXB-AS3 was decreased following transfection with sh-HOXB-AS3-1 and -2 in both cell lines. Subsequently, the viability and proliferation ability of the transfected cells was analyzed, and it was found that cells, in which HOXB-AS3 mRNA expression was knocked down, had reduced viability and proliferation ability from CCK-8 ( $\mathrm{P}<0.001$; Fig. 2C) and colony formation assays $(\mathrm{P}<0.001)$ (Fig. 2D and $\mathrm{E})$, respectively. This indicated that HOXB-AS3 promoted proliferation.

HOXB-AS3 directly binds with IGF2BP2 and thereby stabilizes c-Myc mRNA. HOXB-AS3 was reported to interact with hnRNPA1 and affect the ratio of PKM1/PKM2 (8), and IGF2BP2 was one of the potential interacting proteins (8). The mRNA and protein expression level of IGF2BP2 was analyzed in the cell lines transfected with sh-HOXB-AS3-1 and -2. The expression of IGF2BP2 was rarely changed, the expression between groups showed no significance (Fig. 3A and B). Next Flag-tagged HOXB-AS3 was transfected into the 293T cell line and was then subjected to a Co-IP assay. IGF2BP2 was detected and the results indicated that HOXB-AS3 binds with IGF2BP2 as a whole complex (Fig. 3C). IGF2BP2 is a well-studied m6A reader stabilizing c-Myc mRNA (14); therefore, the protein expression level of c-Myc was analyzed in cells transfected with sh-HOXB-AS3-1 and -2 and the results showed that c-Myc protein expression level was decreased with the knockdown of HOXB-AS3 in both cell lines (Fig. 3D).

Micro-peptide HOXB-AS3, but not HOXB-AS3 RNA promotes the proliferation of the OSCC cells. As aforementioned, it was found that HOXB-AS3 promoted the proliferation and viability of the OSCC cell lines by stabilizing c-Myc mRNA. However, the mRNA and the protein were not separated; therefore, to identify the potential function, the HOXB-AS3 open reading frame was re-expressed in HOXB-AS3 stable knockdown cell lines, the graphic illustration of ORF and HOXB-AS3 was shown in Fig. S2. Subsequently, the protein expression levels of HOXB-AS3 and c-Myc were analyzed (Fig. 4A and B, respectively). The results showed that the protein expression level of c-Myc was restored. In addition, cell viability and 
A

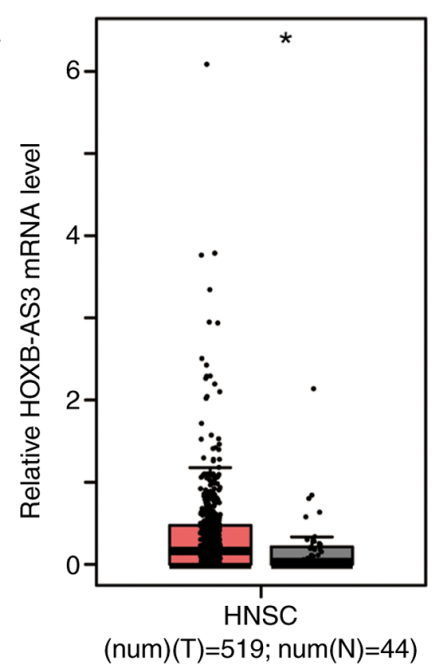

C

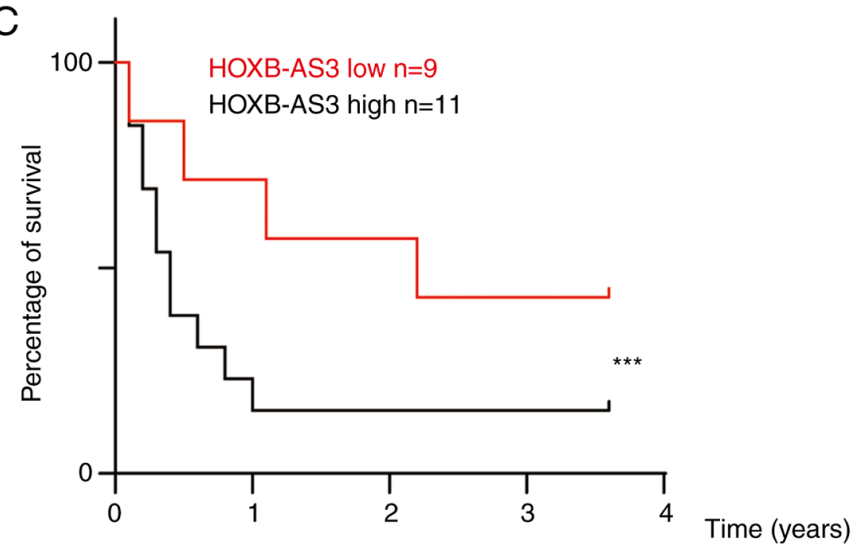

B

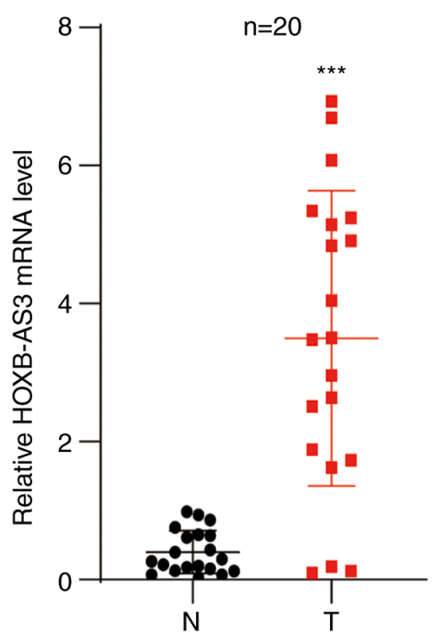

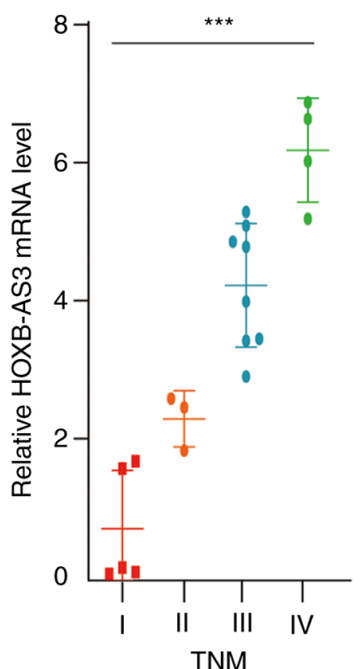

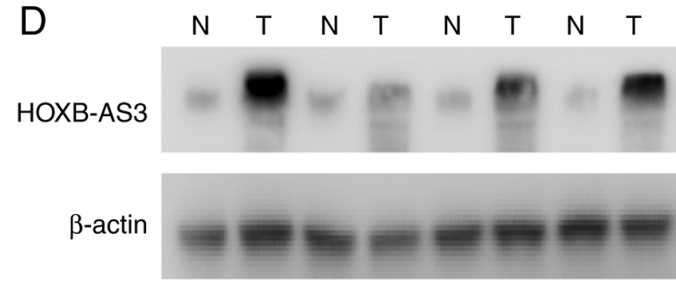

Figure 1. HOXB-AS3 mRNA expression level is upregulated in OSCC tissues and is associated with poor prognosis. The relative mRNA expression level of HOXB-AS3 was analyzed in (A) The Cancer Genome Atlas database and (B) in patients with OSCC recruited into the present study. (C) Patients with OSCC were divided into 2 groups according to the mean mRNA expression level and overall survival was analyzed. (D) The protein expression level of HOXB-AS3 in 4 paired adjacent normal and cancer tissues. ${ }^{*} \mathrm{P}<0.05 .{ }^{* * * *} \mathrm{P}<0.001$. OSCC, oral squamous cell carcinoma; HNSC, head-neck squamous cell carcinoma; $\mathrm{N}$, normal; $\mathrm{T}$, tumor; TNM, tumor-node-metastasis.

proliferation was analyzed and the results also showed an increase in both (Fig. 4C-E), indicating that the HOXB-AS3 protein, but not HOXB-AS3 mRNA exerted its oncogenic function.

\section{Discussion}

OSCC is one of the most common malignancies worldwide (1). Although many risk factors, such as alcohol addiction and HPV infections, have been reported to be involved in the tumourigenesis and progression of OSCC (15), genomic mutations in PTEN and AKT lead to the uncontrolled phosphorylation of AKT and thus promote the proliferation of $\operatorname{OSCC}(16,17)$. Oncogenic amplification, such as in the epidermal growth factor receptor promotes the activation of downstream signaling and eventually promotes OSCC survival (18). However, the exact mechanism of tumourigenesis in OSCC remains unknown.

LncRNAs serve crucial roles in the tumourigenesis and progression of OSCCs (19-24). PDGF-BB regulates the transformation of fibroblasts into cancer-associated fibroblasts via modulating the expression of IncRNA LURAP1L-AS1 and this is mostly accomplished by affecting the NF- $\kappa B$ signaling pathway (19). IncRNA LINC01929 promotes the expression of Forkhead box protein $\mathrm{C} 1$ by sponging the microRNA(miR)-137-3p and serves oncogenic roles (20). Tumor associated macrophages secrete LncRNA LBX1-AS1 via the exosome, inhibiting OSCC progression by targeting miR-182-5p/Forkhead box protein O3 (21). LncRNA PART1 directly binds with the E2-ubiquitin ligating enzyme of EZH2 and promotes cell proliferation (22). Taken together, lncRNAs mostly exert their functions through sponging miRNA and RNA binding protein partner (22). However, lncRNAs are no longer absolutely non-coding. With the application of high-throughput sequencing, an increasing number of IncRNAs have been reported to be able to be translated into proteins (23). HOXB-AS3 is one of these translatable IncRNAs (9). The biological function of HOXB-AS3 varies in colon cancer (9) and lung cancer (24). However, HOXB-AS3 is not only a coding mRNA, but the lncRNA and its encoded protein exert their functions separately $(9,24)$. This may be the reason why conflicting biological functions exist. In most studies, HOXB-AS3 exert its' function as a ceRNA and the biological function was the overall function of ceRNA and HOXB-AS3 protein $(9,24)$. However, in the study which first described HOXB-AS3 protein, it was found that HOXB-AS3 protein exert its' tumor suppressing function by switching the 

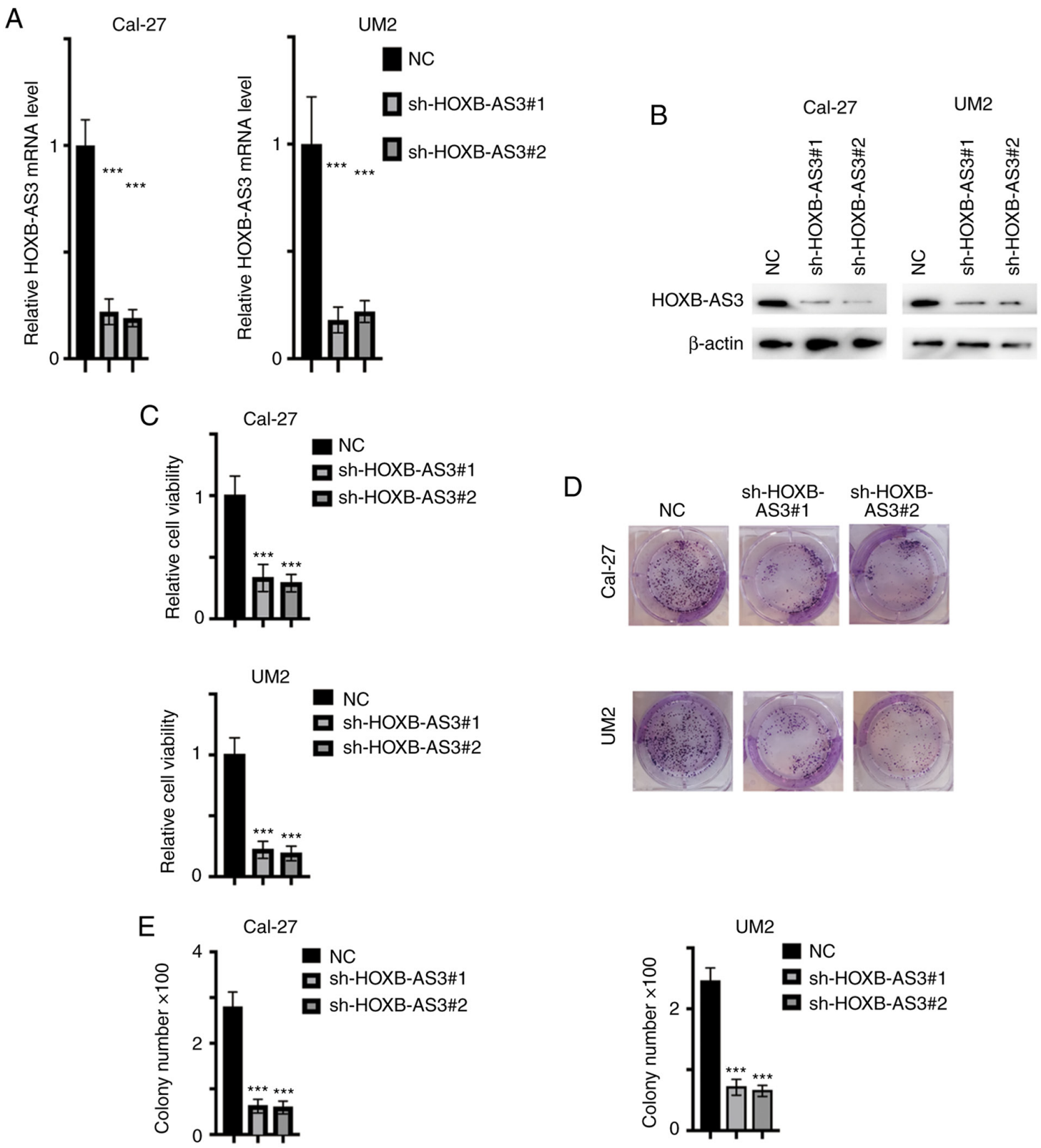

Figure 2. HOXB-AS3 promotes the proliferation and viability of the OSCC cell lines. (A) The OSCC cell lines were transfected with 2 shRNAs or NC and the (A) relative mRNA and (B) protein expression level of HOXB-AS3 was analyzed. (C) The relative cell viability the 2 OSCC cell lines was analyzed following transfection with 2 shRNAs or NC. (D) Representative images of the colony formation assay in the Cal-27 and UM2 cell lines following transfection with 2 shRNAs or NC and the results were (E) statistically analyzed. ${ }^{* * *} \mathrm{P}<0.001$ vs. NC. NC, negative control; sh, short hairpin; OSCC, oral squamous cell carcinoma.

ratio of PKM1/Pyruvate Kinase M1/M2. In the LC/MS mass spectrum analysis performed in the aforementioned study, IGF2BP2 was one of the potential interacting protein (9). IGF2BP2 is a novel $\mathrm{m} 6 \mathrm{~A}$ reader and promotes the stability of mRNAs, such as Myc (9). The present study uncovered the role of HOXB-AS3 in OSCC and to further uncover the mechanism, the IncRNA and protein roles were separated. The present study reexpressed HOXB-AS3 protein in stable knockdown cells and detected the proliferation rate. Proliferation was completely restored with the reexpression of HOXB-AS3 protein in the present study, indicating that in OSCC, HOXB-AS3 promoted tumourigenesis and that this oncogenic role is HOXB-AS3 protein dependent. The findings of the present study indicated that HOXB-AS3 protein, but not the IncRNA exert its' function in OSCC.
The present study analysed the expression pattern of HOXB-AS3 in OSCC and its correlation with the prognosis of patients in the TCGA database and our in-house database, however, the TCGA database showed that the level of HOXB-AS3 didn't predict the prognosis, it may because that the Head and Neck tumor in TCGA database doesn't just contain data for OSCC. We found that HOXB-AS3 overexpression promoted the proliferation of OSCC by stabilizing c-myc mRNA by directly binding to IGF2BP 2 . HOXB-AS3 binds with IGF2BP2 and promotes its' function in stabilizing c-myc. To the best of our knowledge, the present study is the first to have demonstrated the potential biological function and underlying mechanism of HOXB-AS3 in OSCC. A limitation of the present study was the lack of in vivo experiments which should be conducted by future studies to verify the findings 

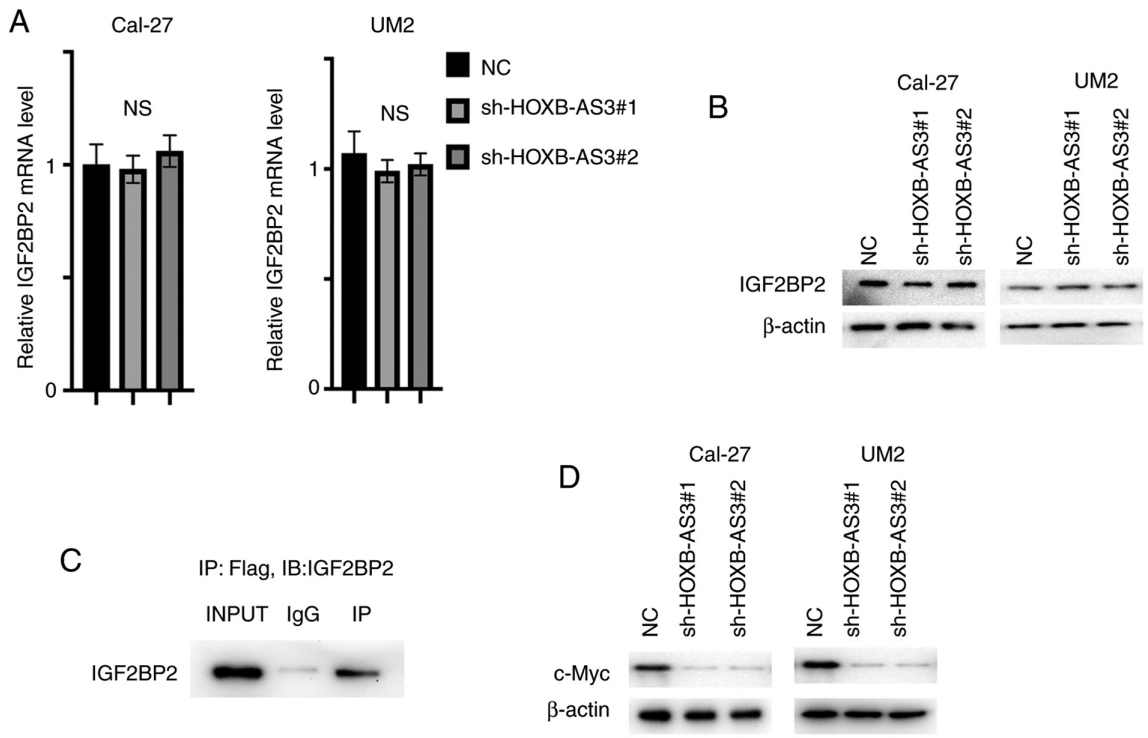

Figure 3. HOXB-AS3 binds with IGF2BP2 and promotes c-Myc stability. The (A) relative mRNA and (B) protein expression level of IGF2BP2 in the Cal-27 and UM2 cell lines following transfection with 2 shRNAs or NC. (C) The 293T cell line was transfected with flag-tagged HOXB-AS3, harvested and subjected to a Co-immunoprecipitation assay using a Flag antibody, followed by western blot analysis to detect IGF2BP2. (D) Western blot of c-Myc in different cell lines following transfection with 2 shRNAs or NC. NC, negative control; sh, short hairpin; NS, non-significant; IP, immunoprecipitate.

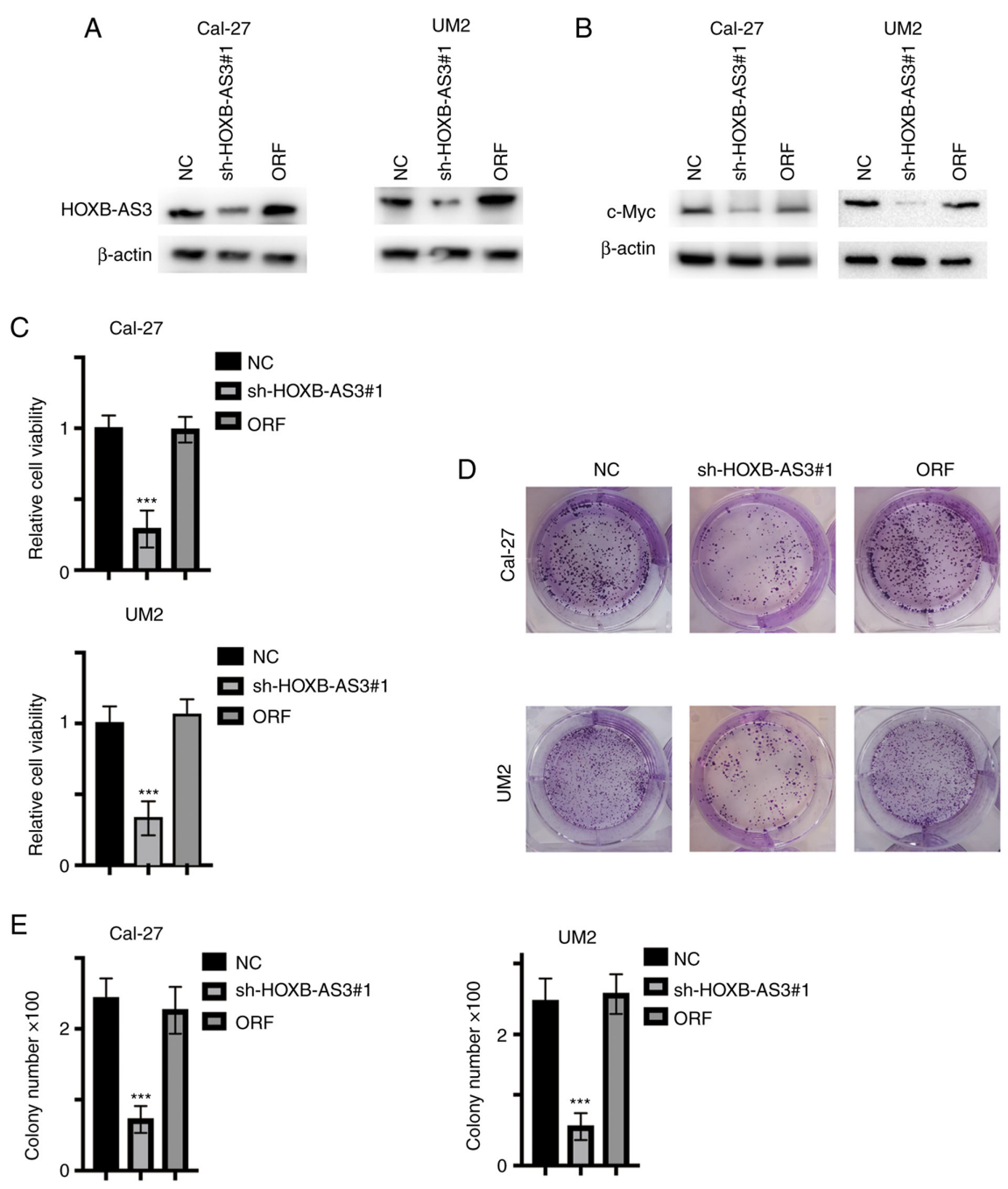

Figure 4. HOXB-AS3 plays an oncogenic role in a HOXB-AS3 protein-dependent manner. The HOXB-AS3 stable knockdown cell lines were transfected with the HOXB-AS3 ORF and the protein expression level of (A) HOXB-AS3 and (B) c-Myc was detected. (C) Relative cell viability in different cell lines transfected with the HOXB-AS3 ORF, shRNA or NC. (D) Representative images of the colony formation assay in the Cal-27 and UM2 cell lines transfected with the HOXB-AS3 ORF, shRNA or NC, and the results were (E) statistically analyzed. ${ }^{* * *} \mathrm{P}<0.001$. ORF, open reading frame; sh, short hairpin; NC, negative control. 
of the present study. Targeting HOXB-AS3 may be one of the potential therapy strategies for patients with OSCC.

\section{Acknowledgements}

The authors would like to thank Professor Wang (Department of Oral and Maxillofacial Surgery, Affiliated Hospital of Jiangxi University of Traditional Chinese Medicine, Nanchang, China) for the support with research design and providing materials.

\section{Funding}

No funding was received.

\section{Availability of data and materials}

All data generated or analyzed during this study are included in this published article.

\section{Authors' contributions}

ZGZ designed the article. FL, YYM, HL, XLL, HC, YZ and ZGZ performed the experiments, data analysis and wrote the manuscript. HC and ZGZ confirm the authenticity of all the raw data. All authors have read and approved the final manuscript.

\section{Ethics approval and consent to participate}

Studies were performed conforming to the Declaration of Helsinki and approval was obtained from the Ethics Committee of the Affiliated Stomatological Hospital of Nanchang University (Jiangxi, China). Clinical samples were collected from patients after written informed consent was obtained.

\section{Patient consent for publication}

Not applicable.

\section{Competing interests}

The authors declare that they have no competing interests.

\section{References}

1. Bray F, Ferlay J, Soerjomataram I, Siegel RL, Torre LA and Jemal A: Global cancer statistics 2018: GLOBOCAN estimates of incidence and mortality worldwide for 36 cancers in 185 countries. CA Cancer J Clin 68: 394-424, 2018.

2. Li P, Fang Q, Yang Y, Chen D, Du W, Liu F and Luo R: Survival significance of number of positive lymph nodes in oral squamous cell carcinoma stratified by p16. Front Oncol 11: 545433, 2021.

3. Antonsson A, de Souza M, Wood ZC, Carroll A, Van K Paterson L, Pandeya N and Whiteman DC: Natural history of oral HPV infection: Longitudinal analyses in prospective cohorts from Australia. Int J Cancer 148: 1964-1972, 2021.

4. Panarese I, Aquino G, Ronchi A, Longo F, Montella M, Cozzolino I, Roccuzzo G, Colella G, Caraglia M and Franco R: Oral and Oropharyngeal squamous cell carcinoma: Prognostic and predictive parameters in the etiopathogenetic route. Expert Rev Anticancer Ther 19: 105-119, 2019.

5. Yang H, Zhang Z, Peng R, Zhang L, Liu H, Wang X, Tian Y and Sun Y: RNA-Seq analysis reveals critical transcriptome changes caused by sodium butyrate in DN mouse models. Biosci Rep 30: BSR20203005, 2021.
6. Yan T, Shen C, Jiang P, Yu C, Guo F, Tian X, Zhu X, Lu S, Han B, Zhong M, et al: Risk SNP-induced IncRNA-SLCC1 drives colorectal cancer through activating glycolysis signaling. Signal Transduct Target Ther 6: 70, 2021.

7. Qin Y, Hou Y, Liu S, Zhu P, Wan X, Zhao M, Peng M, Zeng H, Li Q, Jin T, et al: A novel long non-coding RNA Inc030 maintains breast cancer stem cell stemness by stabilizing SQLE mRNA and increasing cholesterol synthesis. Adv Sci (Weinh) 8: $2002232,2020$.

8. Zhang F, Wang H, Yu J, Yao X, Yang S, Li W, Xu L and Zhao L: LncRNA CRNDE attenuates chemoresistance in gastric cancer via SRSF6-regulated alternative splicing of PICALM. Mol Cancer 20: 6, 2021.

9. Huang JZ, Chen M, Chen D, Gao XC, Zhu S, Huang H, Hu M, Zhu $\mathrm{H}$ and Yan GR: A peptide encoded by a putative lncRNA HOXB-AS3 suppresses colon cancer growth. Mol Cell 68: 171-184. e6, 2017.

10. Xu S, Jia G, Zhang H, Wang L, Cong Y, Lv M, Xu J, Ruan H, Jia X, Xu P and Wang Y: LncRNA HOXB-AS3 promotes growth, invasion and migration of epithelial ovarian cancer by altering glycolysis. Life Sci 264: 118636, 2021.

11. Papaioannou D, Petri A, Dovey OM, Terreri S, Wang E, Collins FA, Woodward LA, Walker AE, Nicolet D, Pepe F, et al: The long non-coding RNA HOXB-AS3 regulates ribosomal RNA transcription in NPM1-mutated acute myeloid leukemia. Nat Commun 10: 5351, 2019.

12. Beltramini GA, Belloni LM, Fusco N, Sacconi A, Muti P, Baj A, Bolzoni AR and Giannì AB: Comparing prognostic utility between the 8th edition of TNM staging system and the lymph node ratio for oral squamous cell carcinoma. Head Neck: Jun 11, 2021 (Epub Ahead of Print).

13. Livak KJ and Schmittgen TD: Analysis of relative gene expression data using real-time quantitative PCR and the 2(-Delta Delta C(T)) method. Methods 25: 402-408, 2001.

14. Huang H, Weng H, Sun W, Qin X, Shi H, Wu H, Zhao BS, Mesquita A, Liu C, Yuan CL, et al: Recognition of RNA $\mathrm{N}^{6}$-methyladenosine by IGF2BP proteins enhances mRNA stability and translation. Nat Cell Biol 20: 285-295, 2018.

15. Cheng J, Zhou X, Feng W, Jia M, Zhang X, An T, Luan M, Pan Y, Zhang S, Zhou Z, et al: Risk stratification by long non-coding RNAs profiling in COVID-19 patients. J Cell Mol Med 25: 4753-4764, 2021

16. Starzynska A, Adamska P, Sejda A, Sakowicz-Burkiewicz M, Adamski $Ł$, Marvaso G, Wychowański P and Jereczek-Fossa BA: Any role of PIK3CA and PTEN biomarkers in the prognosis in oral squamous cell carcinoma? Life (Basel) 10: 325, 2020.

17. Manikandan M, Deva Magendhra Rao AK, Arunkumar G, Manickavasagam M, Rajkumar KS, Rajaraman R and Munirajan AK: Oral squamous cell carcinoma: MicroRNA expression profiling and integrative analyses for elucidation of tumourigenesis mechanism. Mol Cancer 15: 28, 2016.

18. Saravani S, Parsamanesh N and Miri-Moghaddam E: Role of EGFR gene polymorphisms in oral squamous cell carcinoma patients of Southeast Iran: A case-control study. Caspian J Intern Med 11: 391-397, 2020.

19. Ren X, Li L, Wu J, Lin K, He Y and Bian L: PDGF-BB regulates the transformation of fibroblasts into cancer-associated fibroblasts via the lncRNA LURAP1L-AS1/LURAP1L/IKK/I $\kappa / \mathrm{NF}-\kappa \mathrm{B}$ signaling pathway. Oncol Lett 22: 537, 2021.

20. Che H, Che Y, Zhang Z and Lu Q: Long non-coding RNA LINC01929 accelerates progression of oral squamous cell carcinoma by targeting the miR-137-3p/FOXC1 axis. Front Oncol 11: 657876, 2021 .

21. Ai Y, Wei H, Wu S, Tang Z, Li X and Zou C: Exosomal lncRNA LBX1-AS1 derived from RBPJ overexpressed-macrophages inhibits oral squamous cell carcinoma progress via miR-182-5p/FOXO3. Front Oncol 11: 605884, 2021.

22. Yu Q, Du Y, Wang S and Zheng X: LncRNA PART1 promotes cell proliferation and inhibits apoptosis of oral squamous cell carcinoma by blocking EZH2 degradation. J Biochem: Mar 16, 2021 (Epub Ahead of Print).

23. Lu S, Zhang J, Lian X, Sun L, Meng K, Chen Y, Sun Z, Yin X, $\mathrm{Li}$ Y, Zhao J, et al: A hidden human proteome encoded by 'non-coding' genes. Nucleic Acids Res 47: 8111-8125, 2019.

24. Jiang W, Kai J, Li D, Wei Z, Wang Y and Wang W: lncRNA HOXB-AS3 exacerbates proliferation, migration, and invasion of lung cancer via activating the PI3K-AKT pathway. J Cell Physiol 235: 7194-7203, 2020.

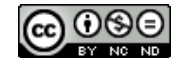

This work is licensed under a Creative Commons Attribution-NonCommercial-NoDerivatives 4.0 International (CC BY-NC-ND 4.0) License. 